\title{
Resonancia religiosa en México: Cifras básicas
}

\author{
Religious Resonance in Mexico: Basic Data
}

Marco Ornelas

Departamento de Ciencias Sociales y Políticas, Universidad Iberoamericana, México

\section{RESUMEN}

El objetivo de esta nota es presentar información de membresía religiosa en México al año 2010, además de proporcionar la información oficial disponible sobre ministros de culto y asociaciones religiosas registrados en el país. Adicionalmente, intenta mostrar una manera en que puede hacerse uso de estadísticas de membresía/adscripción religiosa dentro de la sociología religiosa luhmanniana, a partir del concepto de resonancia religiosa. La resonancia religiosa indica el porcentaje de la población que declara su pertenencia a alguna religión, presuponiendo la existencia de un sistema de comunicaciones religiosas mundializado. A pesar de ser México un país con resonancia religiosa mayoritariamente católica (82.7\%), no menos de 15 orientaciones religiosas distintas a la católica son distinguibles en el territorio nacional. El análisis estadístico presentado permite la sospecha fundada de una inflación de la resonancia católica en México.

PALABRAS ClAVE: Niklas Luhmann; Resonancia (membresía) religiosa; Ministros de culto; Asociaciones religiosas; México

\section{ABSTRACT}

This research note aims to present information on religious membership in Mexico to the year 2010, as well as the official available information on registered cult ministers and religious associations. In addition, it proposes a new way of considering data on religious membership within the sociology of religion of Niklas Luhmann, on the basis of the concept of religious resonance. Religious resonance indicates the population percentage that declares belonging to some denomination, given the assumption of an existing world religious communication system. Even though in Mexico the Catholic resonance is of $82.7 \%$, there exist no less than 15 denominations different from Catholicism. The statistical analysis presented allows for the sound suspicion of an inflated Catholic resonance in Mexico.

KEYWORDS: Niklas Luhmann; Religious Resonance (Membership); Ministers of Cult; Religious Associations; Mexico

\section{INTRODUCCIÓN: EL ESTUDIO SOCIOLÓGICO DE LAS RELIGIONES}

El objetivo de esta nota es presentar información de membresía religiosa en México y proporcionar la información oficial disponible sobre ministros de culto y asociaciones religiosas registrados en el país. Adicionalmente, intenta mostrar una manera en que puede hacerse uso de estadísticas de membresía/adscripción religiosa dentro de la sociología religiosa luhmanniana, a partir del concepto de resonancia religiosa.

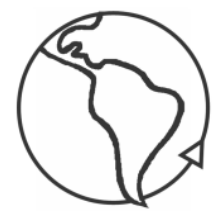

REVISTA MAD - UNIVERSIDAD DE CHILE

Revista del Magíster en Análisis Sistémico Aplicado a la Sociedad

Facultad de Ciencias Sociales, Departamento de Antropología

Universidad de Chile

ISSN 0718-0527

www.revistamad.uchile.cl 
Es importante advertir desde un inicio que esta nota no pretende presentar los conceptos centrales de la sociología de la religión luhmanniana, aunque hará uso de algunas nociones básicas que permitan esclarecer la interpretación particular que se hace aquí de las estadísticas de membresía religiosa. Para aquellos interesados en explorar la sociología de la religión de Niklas Luhmann pueden recurrir a Luhmann (1998, 2007a, 2007b, 2009).

Luhmann se refirió a las religiones exclusivamente como comunicaciones sociales. En sus palabras, cuando se habla de religión se trata "exclusivamente de comunicación religiosa, de sentido religioso que se actualiza en la comunicación como sentido de la comunicación" (Luhmann 2007b: 37).

Las religiones, pues, son consideradas aquí como un fenómeno comunicativo y, por consiguiente, como un suceso difícil de circunscribir en un territorio. Esto quiere decir que las comunicaciones religiosas se relacionan de manera difusa con el espacio. Por ello se hizo necesario plantear un concepto que mostrara esta circunstancia y permitiera darle un sesgo teórico particular a lo que tradicionalmente se consideraría como "membresía/adscripción religiosa". Este concepto es el de resonancia religiosa. La resonancia religiosa indica el porcentaje de la población que declara su pertenencia a alguna religión, ${ }^{1}$ presuponiendo la existencia de un sistema de comunicaciones religiosas mundializado. ${ }^{2}$

La resonancia, en este sentido, implica siempre la interpenetración ${ }^{3}$ (Luhmann 1998: 199-235) entre el sistema mundial de comunicaciones religiosas, representado por las confesiones disponibles para la población en un momento dado, y los sistemas psíquicos de los seres humanos (la facultad consciente, discriminativa, de los pobladores de un territorio). La resonancia religiosa da por supuesta la selección de la población de entre las diversas religiones disponibles en el sistema mundial de comunicaciones religiosas o, dicho de otra manera, la selección de religiones "à la carte" (Luhmann 2007b: 250-251).

\footnotetext{
1 La pregunta en el cuestionario censal es: ¿Cuál es la religión de Juan? Compárese http://www.inegi.org.mx/est/contenidos/proyectos/aspectosmetodologicos/cuestionarios/default.aspx, consultada el 10/06/2014.

${ }^{2}$ Esta sería la consecuencia más obvia e inmediata del uso de este concepto. La otra sería -la más importante por sus implicaciones teóricas- que la resonancia religiosa indica el impacto de las comunicaciones religiosas en el entorno societal interno, esto es, su prestación a otros sistemas sociales. Sobre el concepto de resonancia compárese Luhmann (1989).

${ }^{3}$ Véase Ornelas (2014) para un ejemplo de cómo la comunicación cristiana no conformista interpenetra caso particular de acoplamiento estructural- al iniciador de la reforma protestante, Martín Lutero.
} 


\section{RESONANCIA RELIGIOSA}

La tabla 1 y el gráfico 1 permiten caracterizar el grado de resonancia comunicativa de la religión católica y de religiones distintas a la católica en México. $^{4}$

TABLA 1. RESONANCIA CATÓLICA Y NO CATÓLICA EN MÉXICO, 1950-2010

\begin{tabular}{|c|c|c|c|}
\hline Año & \% católicos & \% no católicos & \% disonante \\
\hline 1950 & 98.2 & 1.8 & 0 \\
\hline 1960 & 96.5 & 2.3 & 0.6 \\
\hline 1970 & 96.2 & 2.2 & 1.6 \\
\hline 1990 & 89.7 & 6.4 & 3.2 \\
\hline 2000 & 88 & 7.6 & 3.5 \\
\hline 2010 & 82.7 & 9.9 & 4.7 \\
\hline
\end{tabular}

FUENTE: Elaboración propia con base en La diversidad religiosa en México. XII Censo general de población y vivienda. INEGI. México, 2005 y de Panorama de las religiones en México 2010. INEG. México, 2011.

GRÁFICO 1. RESONANCIA CATÓLICA Y NO CATÓLICA EN MÉXICO, 1950-2010

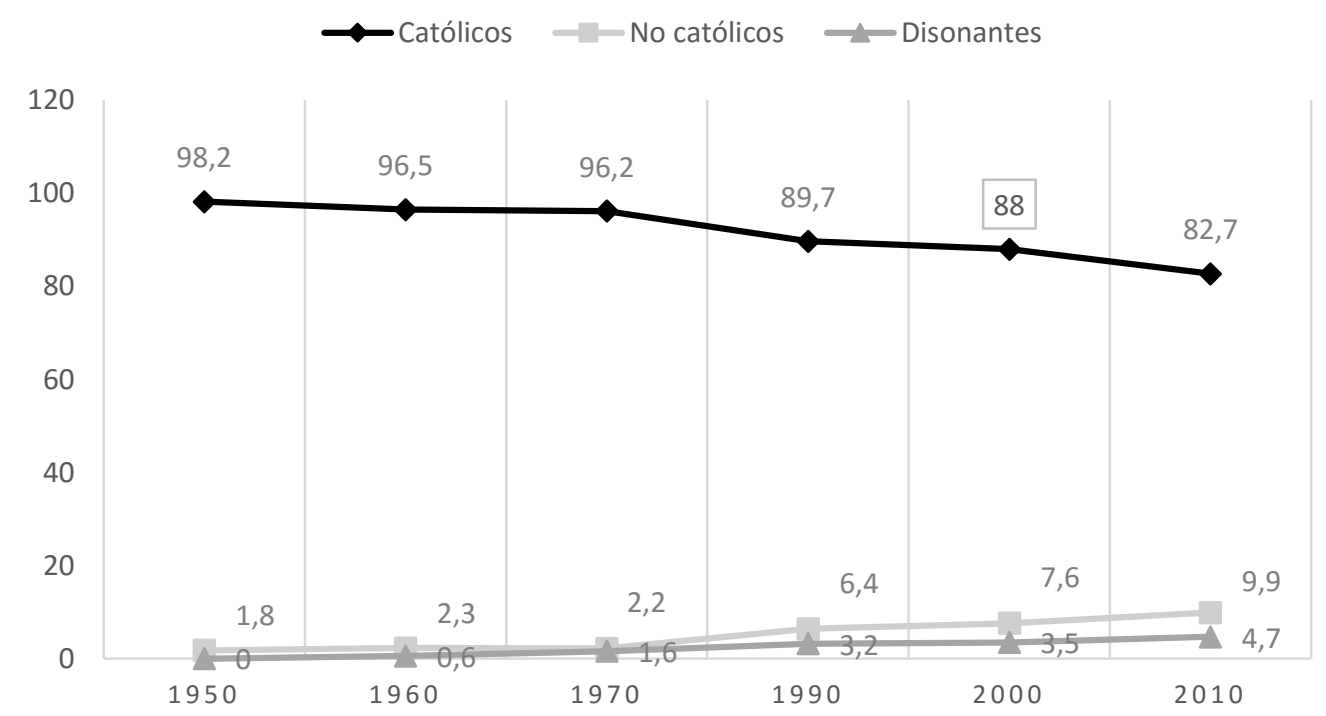

FUENTE: Elaboración propia con base en La diversidad religiosa en México. XII Censo general de población y vivienda. INEGI. México, 2005 y de Panorama de las religiones en México 2010. INEG. México, 2011.

\footnotetext{
${ }^{4}$ Hacia 1910, al inicio de la revolución mexicana y antes del establecimiento de las primeras iglesias pentecostales en el país, Bastian (1990: 138) contabilizó 700 congregaciones protestantes con 70 mil fieles (metodistas, presbiterianos y congregacionalistas). La población total del país era entonces de poco más de 15 millones, por lo que la población protestante como proporción del total no alcanzaba siquiera un medio punto porcentual.
} 
Durante la segunda mitad del siglo XX, México vio disminuir consistentemente la resonancia del catolicismo entre la población (del 98.2\% al 82.7\%), para dar lugar al crecimiento de la resonancia de religiones distintas a la católica (de $1.8 \%$ a 9.9\%). Otro dato de interés: la existencia de un porcentaje visible (4.7\%) de disonancia religiosa, por llamar de algún modo a quienes no profesan fe religiosa alguna.

Estos datos ubican a México dentro de la tendencia predominante de la sociedad mundial hacia la secularización. Por secularización se entiende no tanto la disminuida influencia de la religión en las comunicaciones contemporáneas sino, ante todo, la relación que guarda la religión con la forma de diferenciación social (tipo de sociedad) con primacía en el actual mundo contemporáneo: la sociedad funcionalmente diferenciada o moderna (Luhmann 2007b: 241 y ss.). La sociedad funcionalmente diferenciada o moderna es hoy día la forma de diferenciación social con primado en la sociedad mundial y puede equiparase con la diferenciación de las comunicaciones sociales que ha llevado, entre otras cosas, a la especialización de lenguajes (arte, ciencia, economía, política, religión, intimidad).

¿Existe evidencia de una oferta religiosa múltiple en el país y de tendencias ciertas para un futuro inmediato? La tabla 2, con estadísticos de las principales religiones en México para entidades seleccionadas en los años 2000 y la tabla 3 del 2010, permiten responder afirmativamente esta pregunta. La resonancia religiosa comparada para el último decenio no sólo confirma la caída firme del catolicismo en el país (88 al 82.7\%), sino que permite correlacionar esta disminución con el crecimiento sobre todo de protestantes y evangélicos (5.2 al 7.5\%) aunque también de bíblicos no evangélicos (2 al 2.3\%) y de disonantes (3.5 al 4.7\%), no así de otras religiones que disminuyeron en el período (0.4 al 0.1\%).

El estadístico nacional es utilizado aquí como valor medio ante el cual es posible comparar la resonancia de las distintas comunicaciones religiosas en algunas entidades federativas. Las tablas presentan, además del estadístico nacional, estadísticos para dos de los estados más católicos del país (Guanajuato y Jalisco), para dos de los menos católicos (Chiapas y Tabasco) y para el Distrito Federal (DeFe), Nuevo León, Sonora, Oaxaca, Coahuila, Veracruz, Chihuahua, Tamaulipas y Baja California. Junto a la resonancia católica, en el país existe resonancia de comunicaciones protestantes y evangélicas (menonitas, baptistas, del Nazareno, metodistas, presbiterianos, evangélicos y pentecostales), de comunicaciones bíblicas no evangélicas (testigos de Jehová, adventistas del séptimo día y la iglesia de Jesucristo de los santos de los últimos días [mormones]), y de otras comunicaciones 
religiosas (de origen oriental, judaísmo, islamismo, de raíces étnicas, espiritualista y otras). Este sería, por decirlo de algún modo, el menú de comunicaciones religiosas disponible en el territorio nacional, en espera de ser interpenetradas por la población.

TABLA 2. RESONANCIA RELIGIOSA COMPARADA: 2000

\begin{tabular}{|l|c|c|c|c|c|}
\hline Entidad $^{5}$ & $\begin{array}{c}\text { \% cató- } \\
\text { lica }\end{array}$ & $\begin{array}{c}\text { \% protestante } \\
\text { y evangélica }^{6}\end{array}$ & $\begin{array}{c}\text { \% bíblica no } \\
\text { evangélica }^{7}\end{array}$ & $\begin{array}{c}\text { \% diso- } \\
\text { nante }\end{array}$ & $\begin{array}{c}\text { \% otras } \\
8\end{array}$ \\
\hline Guanajuato (4049950) & 96.4 & 1.3 & 0.7 & 0.7 & 0.1 \\
\hline Jalisco (5541480) & 95.4 & 2 & 0.9 & 0.9 & 0.1 \\
\hline México (11097516) & 91.2 & 3.8 & 1.5 & 1.8 & 0.9 \\
\hline DeFe (7738307) & 90.4 & 3.5 & 1.4 & 2.9 & 1.1 \\
\hline Nacional (84794454) & 88 & 5.2 & 2 & 3.5 & 0.4 \\
\hline Nvo León (3392025) & 87.9 & 6.2 & 2 & 2.8 & 0.1 \\
\hline Sonora (1956617) & 87.8 & 4.9 & 1.8 & 4.3 & 0.1 \\
\hline Coahuila (2018053) & 86.4 & 6.8 & 1.8 & 3.8 & 0.1 \\
\hline Oaxaca (3019103) & 84.8 & 7.7 & 2.3 & 4 & 0.3 \\
\hline Chihuahua (2621057) & 84.6 & 7.1 & 2 & 5.1 & 0.1 \\
\hline Tamaulipas (2427309) & 82.9 & 8.6 & 2.3 & 4.9 & 0.3 \\
\hline Veracruz (6118108) & 82.9 & 6.9 & 3.3 & 5.9 & 0.2 \\
\hline Baja Calif (2010869) & 81.4 & 7.9 & 2.7 & 6.1 & 0.3 \\
\hline Tabasco (1664366) & 70.4 & 13.6 & 5 & 10 & 0.2 \\
\hline Chiapas (3288963) & 63.8 & 13.9 & 8 & 13.1 & 0 \\
\hline
\end{tabular}

FUENTE: Elaboración propia con base en La diversidad religiosa en México. XII Censo general de población y vivienda. INEGI. México, 2005.

TABLA 3. RESONANCIA RELIGIOSA COMPARADA: 2010

\begin{tabular}{|c|c|c|c|c|c|}
\hline Entidad $^{5}$ & $\begin{array}{l}\text { \% cató- } \\
\text { lica }\end{array}$ & $\begin{array}{l}\text { \% protestante } \\
\text { y evangélica }\end{array}$ & $\begin{array}{l}\text { \% bíblica no } \\
\text { evangélica }\end{array}$ & $\begin{array}{l}\% \text { diso- } \\
\text { nante }\end{array}$ & $\%$ otras $^{8}$ \\
\hline Guanajuato (5486372) & 93.8 & 2.6 & 0.9 & 1.4 & 0.04 \\
\hline Jalisco (7350682) & 92 & 3.2 & 1 & 1.7 & 0.3 \\
\hline México (15175862) & 85.4 & 5.6 & 1.6 & 3.2 & 0.3 \\
\hline Nacional (112336538) & 82.7 & 7.5 & 2.3 & 4.7 & 0.1 \\
\hline DeFe (8851080) & 82.5 & 5.4 & 1.4 & 5.5 & 0.5 \\
\hline Nvo León (4653458) & 82.4 & 8.2 & 2 & 4.1 & 0.05 \\
\hline Sonora (2662480) & 82.3 & 7.7 & 2 & 6.5 & 0.06 \\
\hline
\end{tabular}

\footnotetext{
${ }^{5}$ La población total de 5 y más años aparece entre paréntesis

${ }^{6}$ Comprende a las iglesias protestantes históricas (Anabaptistas/Menonitas, Baptistas, del Nazareno, Metodistas, Presbiterianos y otras) y a las iglesias evangélicas (pentecostales y evangélicas)

${ }^{7}$ Comprende a los Testigos de Jehová, a los Adventistas del séptimo día y a la Iglesia de Jesucristo de los santos de los últimos días (mormones)

${ }^{8}$ Comprende a las iglesias de origen oriental, judaísmo, islamismo, raíces étnicas, espiritualista y otras.
} 


\begin{tabular}{|l|c|c|c|c|c|}
\hline Entidad $^{5}$ & $\begin{array}{c}\text { \% cató- } \\
\text { lica }\end{array}$ & $\begin{array}{c}\text { \% protestante } \\
\text { y evangélica }^{6}\end{array}$ & $\begin{array}{c}\text { \% bíblica no } \\
\text { evangélica }^{7}\end{array}$ & $\begin{array}{c}\text { \% diso- } \\
\text { nante }\end{array}$ & \% otras \\
\hline Oaxaca (3801962) & 80.6 & 10.5 & 2.7 & 4.5 & 0.1 \\
\hline Coahuila (2748391) & 80.4 & 10 & 1.9 & 5.5 & 0.04 \\
\hline Veracruz (7643194) & 78.7 & 9.2 & 3.3 & 6.5 & 0.08 \\
\hline Chihuahua (3406465) & 76.4 & 9.5 & 2 & 7.4 & 0.03 \\
\hline Tamaulipas (3268554) & 73 & 12.2 & 2.5 & 6.7 & 0.03 \\
\hline Baja Calif (3155070) & 72 & 11.9 & 3.1 & 10 & 0.1 \\
\hline Tabasco (2238603) & 64.5 & 18.4 & 5.6 & 9.5 & 0.04 \\
\hline Chiapas (4796580) & 58.3 & 19.2 & 8.2 & 12.2 & 0.06 \\
\hline
\end{tabular}

FUENTE: Elaboración propia con base en Panorama de las religiones en México 2010. INEG. México, 2011

¿Existe evidencia de una regionalización de la resonancia religiosa en el país? La respuesta es afirmativa. Mientras que la resonancia católica se aglutina en el centro-occidente del país, la protestante y evangélica se concentran en el sur-sureste del territorio nacional (INEGI 2005: 27, 35, 43; De la Torre \& Gutiérrez 2007: 37). Para el año 2000, los estados fronterizos con los Estados Unidos (Baja California, Sonora, Chihuahua, Coahuila, Nuevo León y Tamaulipas) presentan una resonancia católica por debajo de la media nacional; para 2010 la brecha se ensancha y Baja California, el menos católico, supera ya la marca de los diez puntos porcentuales por debajo de la resonancia católica en el país. Es cierto, la resonancia protestante y evangélica en estados de la frontera norte es mayor al estadístico promedio nacional -la única excepción es Sonora en el año 2000-, aunque el dato no rebasa el cinco por ciento en ningún caso. Finalmente, con la excepción de Nuevo León, desde el año 2000 todas las entidades fronterizas muestran una disonancia religiosa mayor a la nacional. 


\section{GRÁFICO 2. RESONANCIA PROMEDIO, 2010}

católica

$85,0 \%$

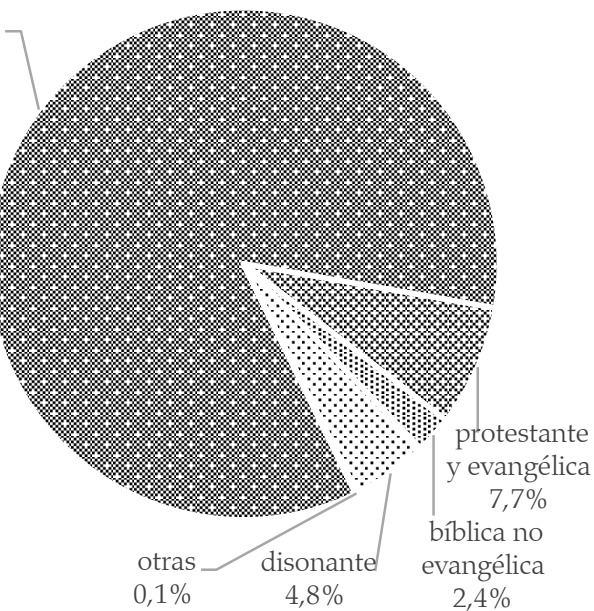

FUENTE: Elaboración propia con base en Panorama de las religiones en México 2010. INEG. México, 2011

\section{GRÁFICO 4. RESONANCIA RELIGIOSA EN CHIAPAS, 2010}

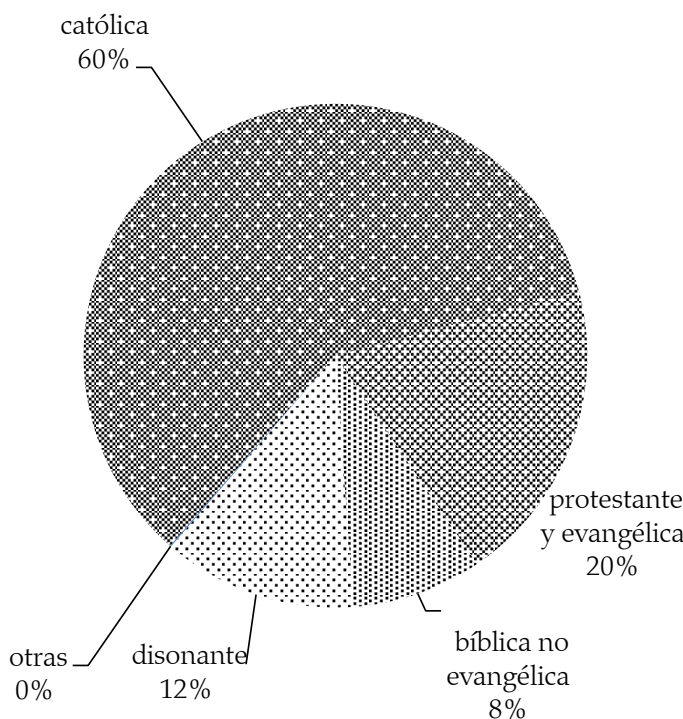

FUENTE: Elaboración propia con base en Panorama de las religiones en México 2010. INEG. México, 2011

\section{GRÁFICO 3. RESONANCIA RELIGIOSA EN GUANAJUATO, 2010}

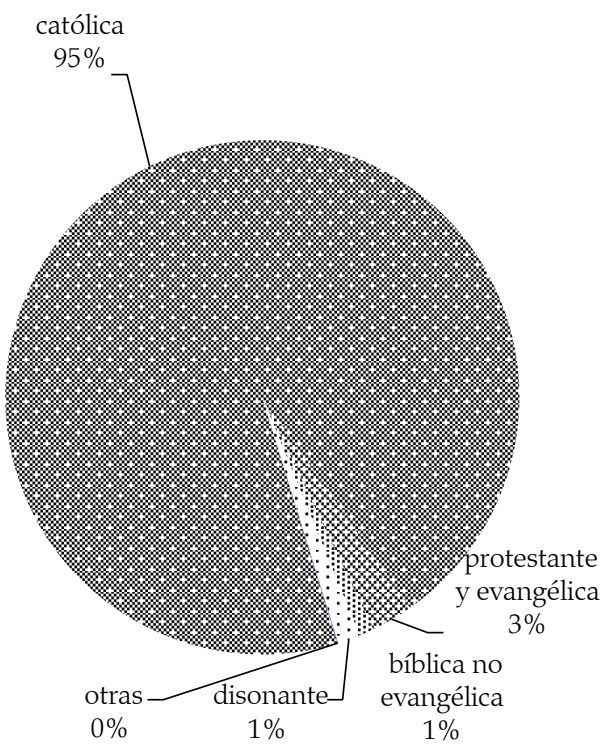

FUENTE: Elaboración propia con base en Panorama de las religiones en México 2010. INEG. México, 2011

\section{GRÁFICO 5. RESONANCIA RELIGIOSA EN EL DISTRITO FEDERAL, 2010}

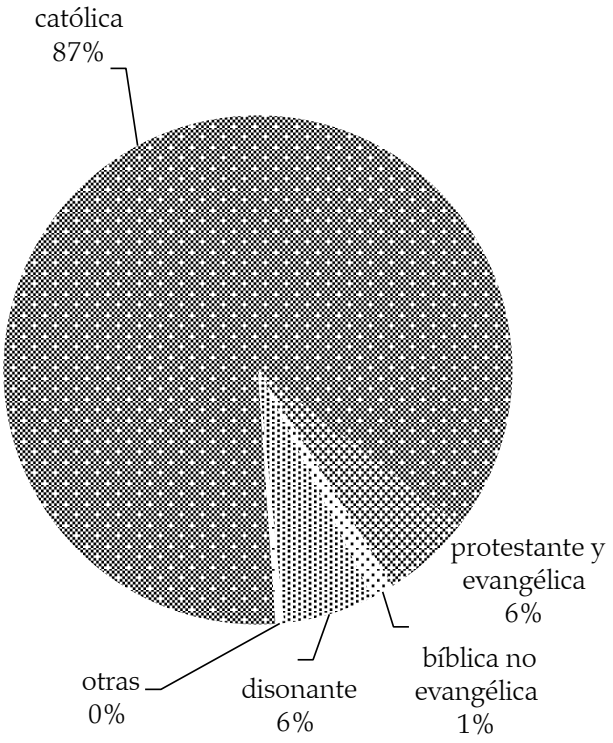

FUENTE: Elaboración propia con base en Panorama de las religiones en México 2010. INEG. México, 2011 
El Distrito Federal se destaca por un dinamismo extremo que concuerda con las tendencias antes apuntadas: además de experimentar un crecimiento notable de protestantes y evangélicos (3.5 al 5.4\%), en el último decenio el catolicismo perdió en la ciudad de México casi 8 puntos porcentuales (90.4 al 82.5\%) para quedar por debajo del estadístico nacional, en tanto que la población disonante creció considerablemente (2.9 a 5.5\%, por arriba del estadístico nacional).

Este es el momento de distinguir entre los distintos protestantismos que hay en México. La tabla 4 y los gráficos que lo acompañan permiten establecer la importancia relativa de la resonancia protestante en México. El protestantismo en México es primero resonancia evangélica y pentecostal -en ese orden-y después resonancia protestante histórica.

TABLA 4. RESONANCIA PROTESTANTE EN ENTIDADES SELECCIONADAS, AÑO 2010

\begin{tabular}{|c|c|c|c|c|c|}
\hline Entidad & $\begin{array}{c}\text { \% Protestan- } \\
\text { te histórica }\end{array}$ & Entidad & $\begin{array}{c}\text { Evangé- } \\
\text { lica }\end{array}$ & Entidad & $\begin{array}{c}\% \text { Pentecos- } \\
\text { tal }^{10} \\
\end{array}$ \\
\hline & $\begin{array}{c}\text { (Nacional }= \\
820744)\end{array}$ & & $\begin{array}{c}\text { (Nacional } \\
=5595116) \\
\end{array}$ & & $\begin{array}{c}\text { Nacional = } \\
1970347) \\
\end{array}$ \\
\hline Chiapas (287945) & 35.1 & $\begin{array}{l}\text { Chiapas } \\
(225935)\end{array}$ & 4 & $\begin{array}{l}\text { Chiapas } \\
(407477)\end{array}$ & 20.7 \\
\hline Veracruz (25212) & 3.1 & $\begin{array}{l}\text { Veracruz } \\
(325535) \\
\end{array}$ & 5.8 & $\begin{array}{l}\text { Veracruz } \\
(351896) \\
\end{array}$ & 17.9 \\
\hline Oaxaca (32696) & 4 & $\begin{array}{l}\text { Oaxaca } \\
(192506) \\
\end{array}$ & 3.4 & $\begin{array}{l}\text { Oaxaca } \\
(174266) \\
\end{array}$ & 8.8 \\
\hline Tabasco (104652) & 12.7 & $\begin{array}{l}\text { Tabasco } \\
(164284) \\
\end{array}$ & 2.9 & $\begin{array}{l}\text { Tabasco } \\
(142341) \\
\end{array}$ & 7.2 \\
\hline Tamps (26967) & 3.3 & $\begin{array}{l}\text { Tamps } \\
(286498) \\
\end{array}$ & 5.1 & $\begin{array}{l}\text { Tamps } \\
(84161)\end{array}$ & 4.3 \\
\hline Baja Calif (14956) & 1.8 & $\begin{array}{l}\text { Baja Calif } \\
(297824)\end{array}$ & 5.3 & $\begin{array}{l}\text { Baja Calif } \\
(65057)\end{array}$ & 3.3 \\
\hline México (22641) & 2.8 & $\begin{array}{l}\text { México } \\
(766978)\end{array}$ & 13.7 & \begin{tabular}{|l|} 
México \\
$(62046)$ \\
\end{tabular} & 3.1 \\
\hline Nvo León (28095) & 3.4 & $\begin{array}{l}\text { Nvo } \\
\text { León } \\
(305855)\end{array}$ & 5.5 & $\begin{array}{l}\text { Nvo León } \\
(48923)\end{array}$ & 2.5 \\
\hline Coahuila (21283) & 2.6 & $\begin{array}{l}\text { Coahuila } \\
(220551) \\
\end{array}$ & 3.9 & $\begin{array}{l}\text { Coahuila } \\
(34304) \\
\end{array}$ & 1.7 \\
\hline Sonora (8936) & 1.1 & $\begin{array}{l}\text { Sonora } \\
(163587)\end{array}$ & 2.9 & \begin{tabular}{|l|} 
Sonora \\
$(31338)$ \\
\end{tabular} & 1.6 \\
\hline
\end{tabular}

\footnotetext{
${ }^{9}$ La población total de las distintas denominaciones protestantes aparece entre paréntesis

${ }^{10}$ Incluye iglesias pentecostales, neopentecostales y de raíces pentecostales (Iglesia del dios vivo, columna y apoyo de la verdad, la luz del mundo)
} 


\begin{tabular}{|c|c|l|c|l|c|}
\hline Entidad & $\begin{array}{c}\text { \% Protestan- } \\
\text { te histórica }\end{array}$ & Entidad & $\begin{array}{c}\text { \% Evangé- } \\
\text { lica }\end{array}$ & Entidad & $\begin{array}{c}\% \text { Pentecos- } \\
\text { tal }^{10}\end{array}$ \\
\hline $\begin{array}{c}\text { (Nacional }= \\
820744)\end{array}$ & $\begin{array}{c}\text { (Nacional } \\
\text { 5595116) }\end{array}$ & $\begin{array}{c}\text { Nacional }= \\
1970347)\end{array}$ \\
\hline DeFe (18062) & 2.2 & $\begin{array}{l}\text { DeFe } \\
(437889)\end{array}$ & 7.8 & $\begin{array}{l}\text { DeFe } \\
(20291)\end{array}$ & 1 \\
\hline Chih (15830) & 1.9 & $\begin{array}{l}\text { Chih } \\
(286981)\end{array}$ & 5.1 & $\begin{array}{l}\text { Chih } \\
(20425)\end{array}$ & 1 \\
\hline
\end{tabular}

FUENTE: Elaboración propia con base en Panorama de las religiones en México 2010. INEG. México, 2011.

GRÁFICO 6. RESONANCIA PROTESTANTE EN MÉXICO, 2010

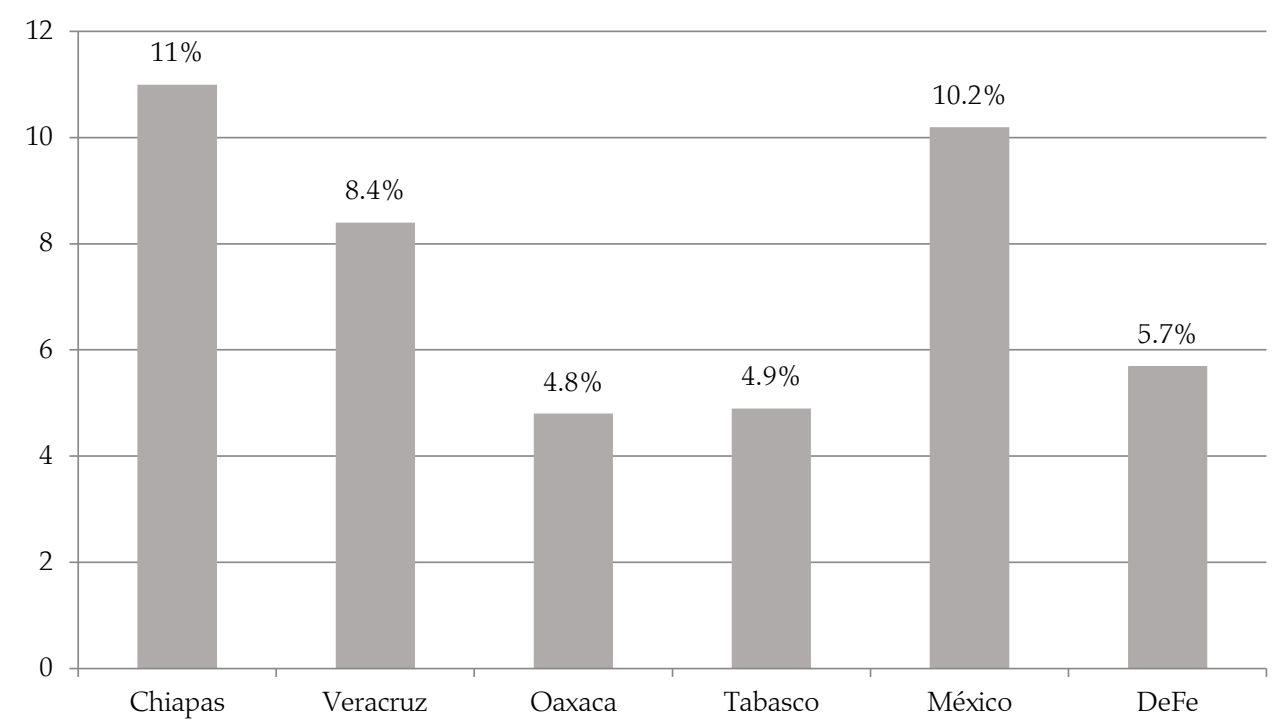

FUENTE: Elaboración propia con base en Panorama de las religiones en México 2010. INEG. México, 2011.

Las iglesias "evangélicas", "cristianas" o "pentecostales" no se refieren a una denominación, "sino a una constelación de grupos identificados doctrinalmente entre sí, pero no adscritos formalmente a una misma organización" (INEGI 2005: 18 y s). Lo que les identifica es la afinidad doctrinal con el protestantismo histórico y una fragmentación organizacional extrema que roza en la autarquía. La distinción entre evangélicos y pentecostales es equívoca (Gutiérrez \& De la Torre 2007: 93) debido a que entre ellos se hacen llamar evangélicos, sobre todo para resaltar un sentido de unidad frente a los católicos "adoradores de imágenes". ${ }^{11}$ También lo es porque el

\footnotetext{
${ }^{11}$ La laxitud del término "evangélicos" permite a Garma $(2007,80)$ afirmar que los pentecostales representan "las asociaciones religiosas no católicas con el mayor número de creyentes en el país".
} 
nombre de muchas de estas agrupaciones puede no incluir la denominación pentecostal o "pentecostés", como en algunos casos ellos mismos prefieren nombrarse (Oaxaca).

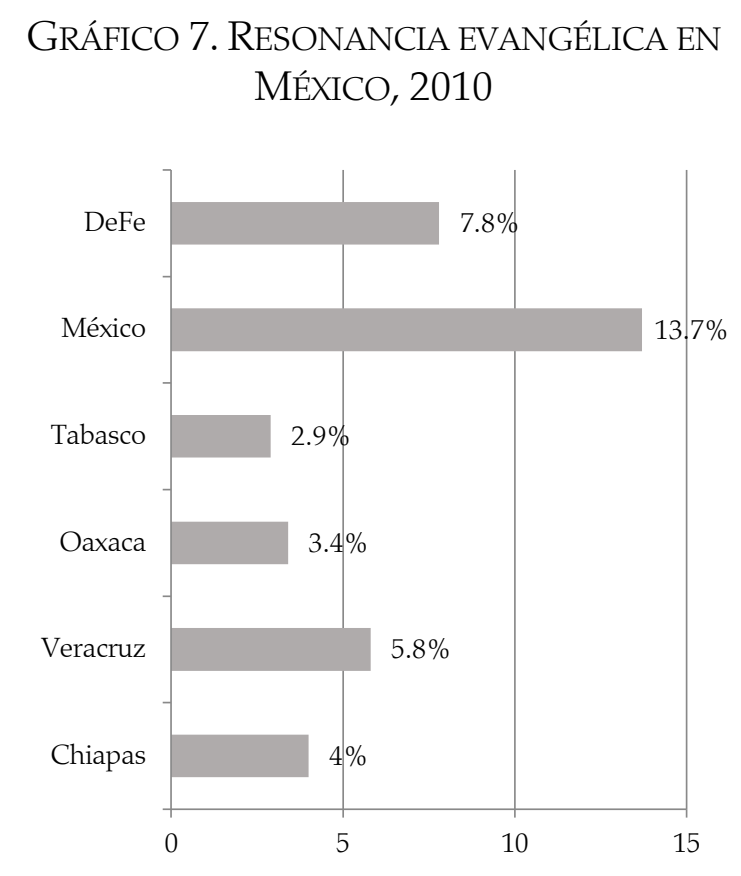

FUENTE: Elaboración propia con base en Panorama de las religiones en México 2010. INEG. México, 2011.

\section{GRÁFICO 8. RESONANCIA PENTECOSTAL EN MÉXICO, 2010}

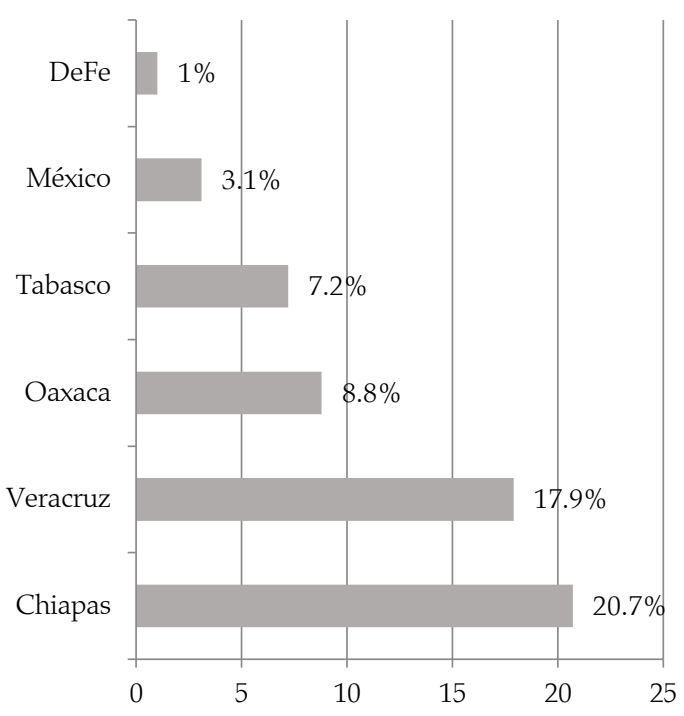

FUENTE: Elaboración propia con base en Panorama de las religiones en México 2010. INEG. México, 2011.

GRÁFICO 9. RESONANCIA PROTESTANTE HISTÓRICA EN MÉXICO, 2010

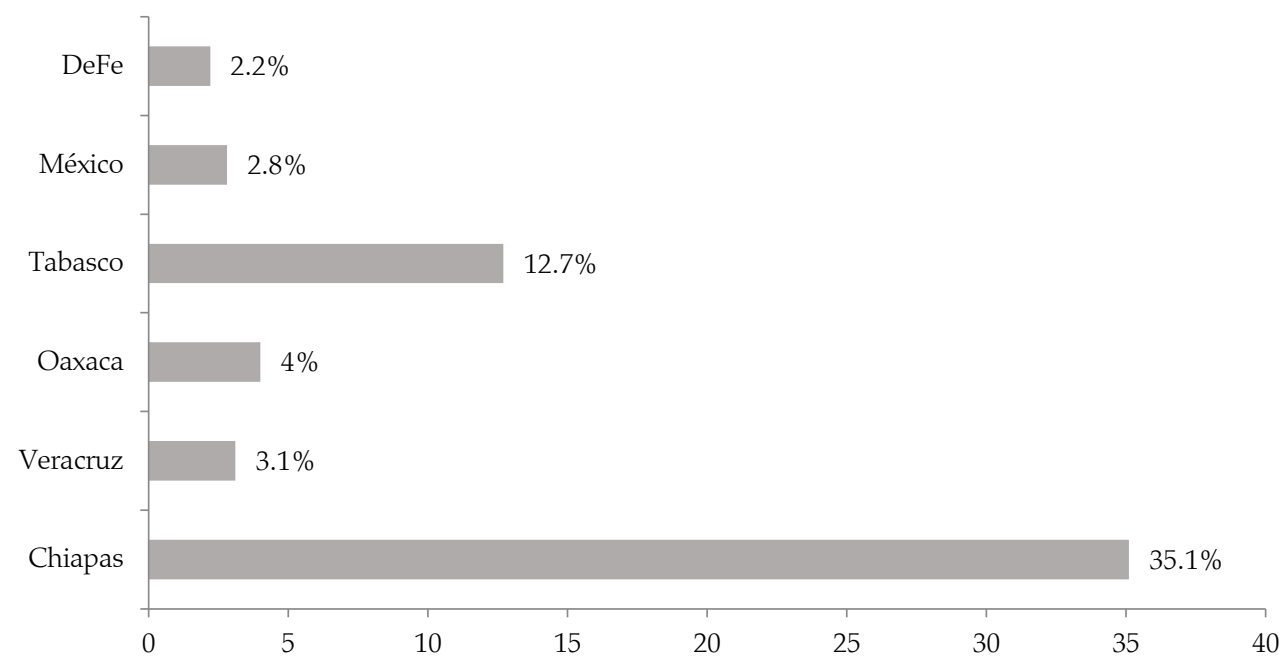

FUENTE: Elaboración propia con base en Panorama de las religiones en México 2010. INEG. México, 2011. 
El protestantismo histórico, por su parte, es la corriente del cristianismo que agrupa a "las iglesias cercanas a la Reforma Religiosa del siglo XVI, movimiento que provocó la separación entre la iglesia católica y las iglesias reformadas" (INEGI 2005: 16 y s). Estas sociedades protestantes tienen presencia en el país desde el siglo xix (Bastian 1990, Gutiérrez 2007: 50). La agrupación de las comunicaciones protestantes utilizada por el INEG -existen protestantes históricos y evangélicos, y de entre estos últimos algunos denominados pentecostales o pentecosteses- está fuertemente influenciada por los estudios de Jean-Pierre Bastian (2006) sobre el protestantismo mexicano.

La tabla 4 presenta información sobre la resonancia de las comunicaciones específicamente protestantes en el país. Las columnas del cuadro muestran la importancia de estados seleccionados en la distribución de los protestantismos mexicanos: histórico, evangélico y pentecostal. Puede decirse, por ejemplo, que Chiapas y Tabasco concentran el $47.8 \%$ de la resonancia protestante histórica en el país (la mayoría del tipo presbiteriano). En Oaxaca, con 4\% de la resonancia protestante histórica del país, predomina la rama baptista (INEGI 2005: 16). El estado de México, el de mayor resonancia evangélica en México, concentra el 13.7\% de evangélicos en el país (INEGI 2005: 18). Los estados fronterizos del norte del país concentran más de una cuarta parte de la resonancia evangélica en México (27.8\%), misma que no hay que enfatizar demasiado puesto que estos mismos estados concentran apenas el $23.4 \%$ de la población protestante y evangélica en el país. Tamaulipas y Baja California se destacan por tener una importante concentración de resonancia pentecostal aunque todavía por debajo de Chiapas, Veracruz, Oaxaca y Tabasco.

Por último, los tres estados con mayor número de pentecostales (Chiapas, Veracruz y Oaxaca) concentran casi la mitad (más del 47\%) de la resonancia pentecostal mexicana. ${ }^{12}$ Chiapas solo concentra más de una tercera parte de los protestantes históricos en el país (35.1\%), y es el estado con menor resonancia católica (58.3\%) y, por tanto, con la mayor resonancia protestante a secas en México (11\%). Según estos datos, la "protestantización" del sur-sureste del país significa "pentecostalización" claramente en los casos de Chiapas, Veracruz y Oaxaca. A nivel nacional la protestan-

\footnotetext{
${ }^{12}$ Coincidentemente, estos mismos tres estados -aunque en distinto orden de importancia: Chiapas, Oaxaca y Veracruz- concentran casi todos los municipios del país con resonancia católica minoritaria (De la Torre y Janssen 2007, 124) en donde habita población mayoritariamente indígena (Garma y Hernández 2007, 203).
} 
tización está mejor representada por Chiapas, que tiene la menor resonancia católica del país. De nuevo, la protestantización de México significa primacía de la resonancia evangélica y, sólo después, creciente importancia de la resonancia pentecostal. ${ }^{13}$

\section{MiNISTROS DE CULTO Y ASOCIACIONES RELIGIOSAS.}

Existe un mayor número de ministros de culto protestantes y evangélicos registrados ante la autoridad gubernamental mexicana -la Dirección General de Asociaciones Religiosas de la SEGOB- mayoría que no guarda proporción con los estadísticos de resonancia religiosa en el país. Esto se muestra claramente en la tabla 5. En 2014, frente a una resonancia católica en el país de no menos del 80\%, los sacerdotes católicos con registro no son siquiera una cuarta parte del total (23.1\%); en cambio, no más del 10\% de resonancia protestante y evangélica se corresponde con casi la mitad de los ministros registrados $(48.3 \%)$.

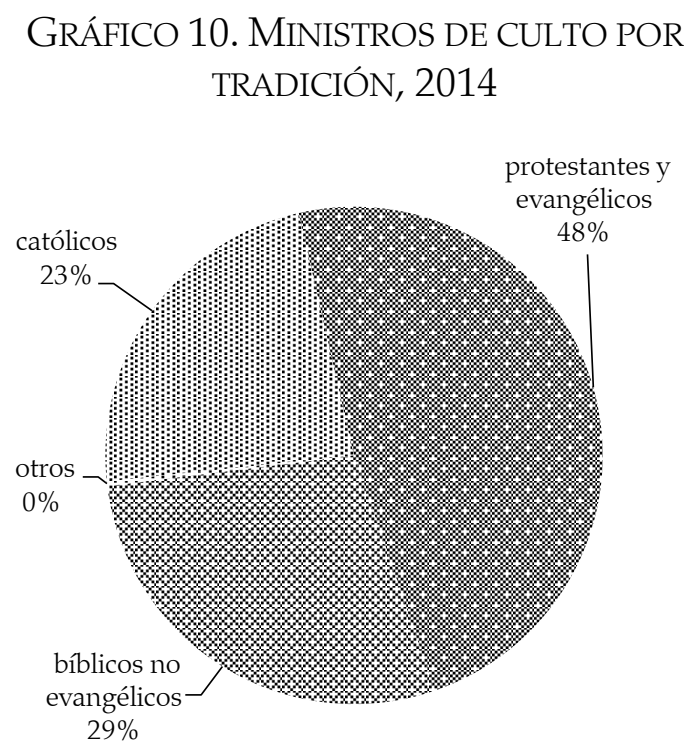

FUENTE: Elaboración propia con datos tomados como se indica

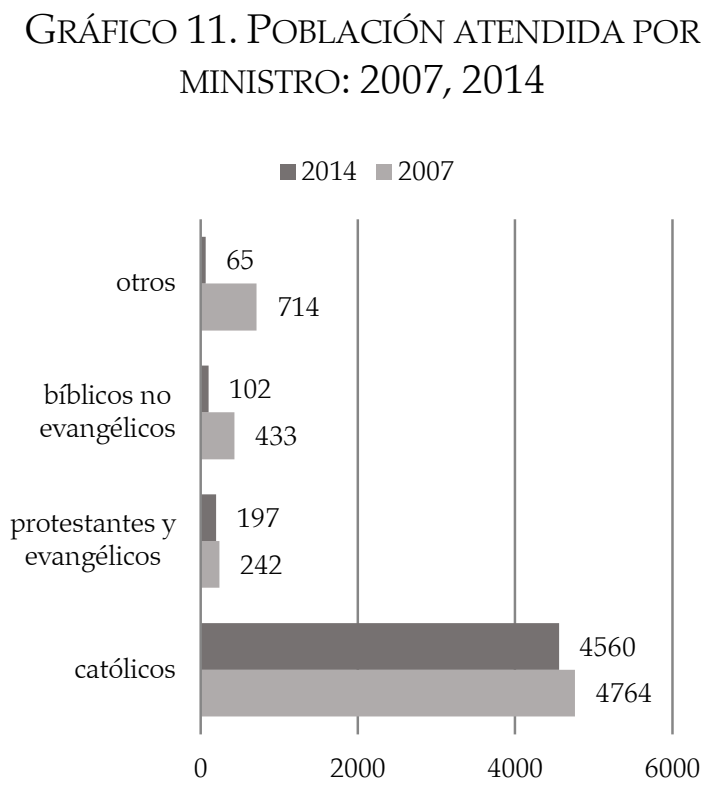

FUENTE: Elaboración propia a partir de la tabla 5 (Ministros de culto con registro: 2007, 2014) y del Panorama de las religiones en México 2010. INEG. México, 2011.

\footnotetext{
${ }^{13}$ Hernández (2007) es más enfático que Garma (véase la n. 4) al sostener que los pentecostales rebasan en número a los evangélicos, aunque esta afirmación no puede sostenerse con la información oficial mostrada en la tabla 4 . Los porcentajes de resonancia que proporciona: 64\% pentecostal, $25 \%$ evangélica y $11 \%$ protestante histórica (contra el 23\%, 67\% y 10\%, respectivamente).
} 
TABLA 5. MinistROS DE CULTO CON REGISTRO: 2007, 2014

\begin{tabular}{|c|c|c|c|c|}
\hline Total nacional & \% católicos & $\begin{array}{c}\text { \% protestantes y } \\
\text { evangélicos }\end{array}$ & $\begin{array}{c}\text { \% bíblicos no } \\
\text { evangélicos }^{14}\end{array}$ & \% otros $^{15}$ \\
\hline $2007=60392^{16}$ & 32.3 & 57.4 & 9.7 & 0.4 \\
\hline $2014=88219^{17}$ & 23.1 & 48.3 & 28.3 & 0.3 \\
\hline
\end{tabular}

FUENTE: Elaboración propia con datos tomados como se indica

Los requisitos impuestos por las distintas iglesias a sus candidatos a ministros de culto puede explicar esto en parte. El sacerdocio católico, por ejemplo, exige al menos el equivalente a estudios de nivel universitario, aunque también pudiera explicarse por la simple inflación de la resonancia católica - por tratarse de la religión tradicional del México contemporáneo, de la religión impuesta por el conquistador-y por la crisis vocacional que experimenta la carrera sacerdotal. Sea como fuere, el caso es que la población atendida por ministro de culto registrado es en verdad contrastante: 4 mil 560 católicos por sacerdote contra únicamente 197 protestantes por pastor, como lo muestra la tabla 6 (datos para 2014).

Tabla 6: Población atendida por ministro: 2007, 2014

\begin{tabular}{|c|c|c|c|c|}
\hline Año & católicos & protestantes y evangélicos & bíblicos no evangélicos & otros \\
\hline 2007 & 4764 & 242 & 433 & 714 \\
\hline 2014 & 4560 & 197 & 102 & 65 \\
\hline
\end{tabular}

FUENTES: Elaboración propia a partir del Cuadro 4 (Ministros de culto con registro: 2007, 2014) y del Panorama de las religiones en México 2010. INEG. México, 2011.

La tabla 7 presenta los datos de asociaciones religiosas con registro para los años 1998 y 2014. De nuevo aquí contrastan los datos de asociaciones religiosas registradas con los datos de resonancia religiosa en el país. Para 2014, no menos de un $80 \%$ de la población mexicana que se dice católica se agrupa en tan solo el $41 \%$ de las asociaciones registradas en el país (3 mil 223 asociaciones), lo que supone el apilamiento promedio de $28 \mathrm{mil}$ 831 católicos por asociación. Como sucede con los datos de población atendida por ministro, lo menos que puede suponerse es una inflación de la

\footnotetext{
${ }^{14}$ Incluye a testigos de Jehová, adventistas y mormones

15 Incluye a orientales (hinduístas, budistas, krishnas), judíos, cristianos ortodoxos, islámicos y pertenecientes a nuevas expresiones

${ }_{16}$ Datos tomados de Ministros registrados por credo religioso. www.asociacionesreligiosas.gob.mx Consultada el 3 de septiembre de 2007.

17 Datos tomados de Ministros de culto por tradición al 2 de mayo de 2014. www.asociacionesreligiosas.gob.mx/es/AsociacionesReligiosas/Numeralia Consultada el 20 de mayo de 2014.
} 
resonancia católica o la notable flexibilidad del compromiso religioso entre católicos -compatible con la secularización creciente de la sociedad mundial-, equiparable a la fidelidad con que la afición se dice partidaria de algún equipo de futbol. La centralización extrema de la organización católica, de tipo piramidal, también puede contribuir a esta situación.

TABLA 7. ASOCIACIONES RELIGIOSAS CON REGISTRO: 1998, 2014

\begin{tabular}{|c|c|c|c|c|}
\hline Total nacional & \% católicos & $\begin{array}{c}\text { \% protestantes } \\
\text { y evangélicos }\end{array}$ & $\begin{array}{c}\text { \% bíblicos no } \\
\text { evangélicos }\end{array}$ & ${\text { \% } \text { otros }^{19}}^{18}$ \\
\hline $1998=5266^{20}$ & 19 & 48.4 & n.e. & $32.6^{21}$ \\
\hline $2014=7976^{22}$ & 40.9 & 58.1 & 0.2 & 0.8 \\
\hline
\end{tabular}

FUENTE: Elaboración propia con datos tomados como se indica

GRÁFICO 12. ASOCIACIONES RELIGIOSAS POR TRADICIÓN, 2014

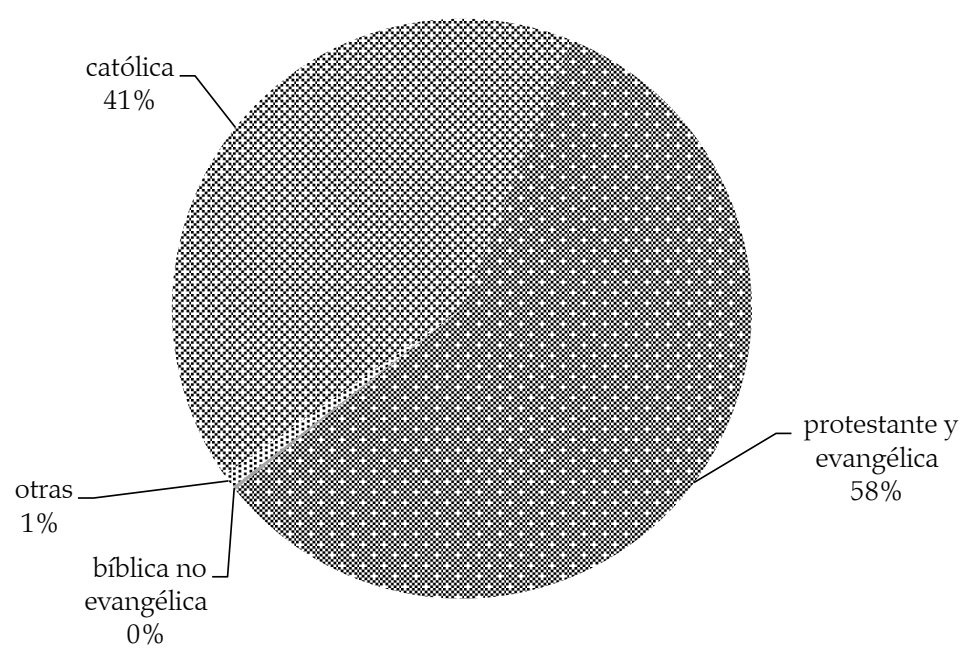

FUENTE: Elaboración propia con datos tomados como se indica

\footnotetext{
${ }^{18}$ Incluye las pertenecientes a los testigos de Jehová, adventistas y mormones

19 Incluye a orientales (hinduístas, budistas, krishnas), judías, cristianas ortodoxas, islámicas y pertenecientes a nuevas expresiones

${ }^{20}$ Datos tomados de Garma (1999: 138)

${ }^{21}$ Incluyen las pertenecientes a bíblicos no evangélicos, para las que no se tiene información

22 Datos tomados de Asociaciones religiosas por tradición al 2 de mayo de 2014. www.asociacionesreligiosas.gob.mx/es/AsociacionesReligiosas/Numeralia. Consultada el 20 de mayo de 2014.
} 


\section{CONCLUSIONES}

A pesar de ser México un país con resonancia religiosa mayoritariamente católica (82.7\%), no menos de 15 orientaciones religiosas distintas a la católica son distinguibles en el territorio nacional. La resonancia no católica alcanza casi el 10\% de la población y la disonancia religiosa cerca del 5\% de los habitantes. Las comunicaciones protestantes y evangélicas son las principales competidoras de las comunicaciones católicas en el país. La resonancia religiosa tiene una clara expresión regional: mientras la resonancia católica se concentra en el centro-occidente, la protestante y evangélica se concentran en el sur-sureste del territorio.

Chiapas, México y Veracruz son los estados con más protestantes del país. El estado de México es el estado con mayor resonancia evangélica (13.7\%), mientras que Chiapas es el de menor resonancia católica (58.3\%) y el de mayor resonancia protestante histórica y pentecostal en México (35.1\%, del tipo presbiteriano, y 20.7\%, respectivamente). Chiapas, Veracruz y Oaxaca concentran la mayor resonancia pentecostal. La protestantización del país sigue significando primero predominancia de la resonancia evangélica y, sólo después, creciente importancia de la resonancia pentecostal. Los estados fronterizos del norte tienen una importante concentración de resonancia evangélica que alcanza el 27.8\% del total. Pudiera decirse que mientras más se avanza al norte en la geografía nacional y se dejan atrás los territorios con alta concentración poblacional india, el protestantismo adquiere una tonalidad más racional y menos mágica y patriarcal.

Contrario a los datos de resonancia religiosa en el país, el $48.3 \%$ del total de ministros de culto registrados son protestantes y evangélicos, contra sólo un $23.1 \%$ de sacerdotes católicos. Es así que la población atendida por sacerdote es de poco más de 4 mil 500 personas, contra sólo 197 hermanos por pastor protestante. Cosa parecida sucede con las asociaciones religiosas registradas, donde menos de la mitad de las asociaciones religiosas registradas son católicas (40.9\%), que dicen atender a cuando menos el $80 \%$ de la población. Los datos permiten suponer la inflación llana de la resonancia católica en México.RM

\section{REFERENCIAS}

Bastian, J. (1990). Las sociedades protestantes y la oposición a Porfirio Díaz en México, 1877-1911. En J. Bastian (Comp.), Protestantes, liberales y francmasones: Sociedades de ideas y modernidad en América Latina, siglo xix (pp. 132-164). México DF: CEHILA/Fondo de Cultura Económica. 
Bastian, J. (2006). De los protestantismos históricos a los pentecostalismos latinoamericanos: Análisis de una mutación religiosa. Revista de Ciencias Sociales (CI), 16, 38-54.

De la Torre, R. \& Gutiérrez, C. (2007). Territorios de la diversidad religiosa hoy. En Atlas de la diversidad religiosa en México (pp. 35-37). México: El Colegio de Jalisco/ El Colegio de la Frontera Norte/ CIESAS/ El Colegio de Michoacán/ Secretaría de Gobernación/ Universidad de Quintana Roo.

De la Torre, R. \& Janssen, E. (2007). Ubicación y patrones de distribución. En Atlas de la diversidad religiosa en México (pp. 124-136). México: El Colegio de Jalisco/ El Colegio de la Frontera Norte/ CIESAS/ El Colegio de Michoacán/ Secretaría de Gobernación/ Universidad de Quintana Roo.

Dirección General de Asociaciones Religiosas (Secretaría de Gobernación) (2007). Directorio de Asociaciones Religiosas (actualizado al 31 de julio). 871 pp. Distribución de ministros registrados por credo religioso (Documento sin título) 2 pp. Ministros de culto registrados al 30 de junio de 2007. 1446 pp. http://www.asociacionesreligiosas.gob.mx> 09/03/2007.

Garma, C. (1999). La situación legal de las minorías religiosas en México: Balance actual, problemas y conflictos. Alteridades, 9(18), 135-144.

Garma, C. (2007). El pentecostalismo. En Atlas de la diversidad religiosa en México (pp. 79-84). México: El Colegio de Jalisco/ El Colegio de la Frontera Norte/ CIESAS/ El Colegio de Michoacán/ Secretaría de Gobernación/ Universidad de Quintana Roo.

Garma, C \& Hernández, A. (2007). Los rostros étnicos de las adscripciones religiosas. En Atlas de la diversidad religiosa en México (pp. 203-226). México: El Colegio de Jalisco/ El Colegio de la Frontera Norte/ CIESAS/ El Colegio de Michoacán/ Secretaría de Gobernación/ Universidad de Quintana Roo.

Gutiérrez, C. (2007). El protestantismo histórico. En Atlas de la diversidad religiosa en México (pp. 50-60). México: El Colegio de Jalisco/ El Colegio de la Frontera Norte/ CIESAS/ El Colegio de Michoacán/ Secretaría de Gobernación/ Universidad de Quintana Roo.

Gutiérrez, C. y De la Torre, R. (2007). Otras evangélicas. En Atlas de la diversidad religiosa en México (pp. 92-97). México: El Colegio de Jalisco/ El Colegio de la Frontera Norte/ CIESAS/ El Colegio de Michoacán/ Secretaría de Gobernación/ Universidad de Quintana Roo.

Hernández, A. (2007). El cambio religioso en México: crecimiento y auge del pentecostalismo. En C. Rivera \& E. Juárez (Eds.), Más allá del espíritu. Actores, acciones y prácticas en iglesias pentecostales (pp. 53-90). México: CIESAS/El Colegio de Michoacán. México.

Instituto Nacional de Estadística, Geografía e Informática (INEGI) (2005). La diversidad religiosa en México. XII Censo general de población y vivienda 2000. México: INEGI.

Instituto Nacional de Estadística, Geografía e Informática (INEGI) (2011). Panorama de las religiones en México 2010. México: INEG.

Luhmann, N. (1989). Ecological Communication. Chicago: The University of Chicago Press.

Luhmann, N. (1998). Sistemas sociales. Lineamientos para una teoría general. Madrid: Anthropos/UIA/CEJA.

Luhmann, N. (2007a). La sociedad de la sociedad. México DF: Herder/UIA. 
Luhmann, N. (2007b). La religión de la sociedad. Madrid: Trotta.

Luhmann, N. (2009). Sociología de la religión. México DF: Herder/UIA.

Ornelas, M. (2014). Lutero y el luteranismo: El buzón postal Martín Lutero. Revista MadUniversidad de Chile, 31, 46-59.

\section{SOBRE EL AUTOR}

Marco Ornelas es Becario Posdoctoral adscrito al Departamento de Ciencias Sociales y Políticas de la Universidad Iberoamericana, México. Doctor en Ciencias Sociales por El Colegio de Sonora, México. Entre sus líneas de trabajo se cuentan: sociología de la religión, teorías sociológicas de la comunicación, historia del cristianismo. Entre sus últimas publicaciones se destacan: La importancia de las herejías para la conformación de una dogmática cristiana [En I. Farías \& J. Ossandón (eds.), Comunicaciones, semánticas y redes. Usos y desviaciones de la sociología de Niklas Luhmann, México D.F., 2011], La sociología de la religión de Niklas Luhmann [En Niklas Luhmann: Sociología de la religión, México D.F., 2009. Autor del prefacio y compilador del libro], The Catholic Mass in a Secular World [Journal of Dharma, 2007].

\section{CONTACTO}

Universidad Iberoamericana, Ciudad de México,

Departamento de Ciencias Sociales y Políticas.

Prol. Paseo de la Reforma 880. Lomas de Santa Fe.

C. P. 01219

México, D. F.

México

marcornelas@hotmail.com

Recibido: Julio 2015

Aceptado: Agosto 2015 